\title{
Validation of a new test that assesses functional performance of the upper extremity and neck (FIT-HaNSA) in patients with shoulder pathology
}

\author{
Joy C MacDermid*1,2, Myriam Ghobrial2, Karine Badra Quirion²,7, \\ Mélanie St-Amour ${ }^{2}$, Tanya Tsui ${ }^{2,3}$, Dave Humphreys ${ }^{2,4}$, John McCluskie ${ }^{2,5}$, \\ Eddy Shewayhat ${ }^{2,6}$ and Vickie Galea ${ }^{2}$
}

\begin{abstract}
Address: ${ }^{1}$ Clinical Research Lab, Hand and Upper Limb Centre, St. Joseph's Health Centre, London, Ontario N6A 4L6, Canada, ${ }^{2}$ School of Rehabilitation Science, McMaster University, Hamilton, Ontario L8S 1C7, Canada, ${ }^{3}$ Heart Lake Sports Medicine \& Orthopedic Clinic, Brampton, Ontario L6Z 1Y4, Canada, ${ }^{4}$ Fowler Kennedy Sport Medicine Clinic, London, Ontario N6A 3N7, Canada, ${ }^{5}$ Hamilton General Hospital, Hamilton Health Sciences, Hamilton, Ontario L8L 2X2, Canada, ${ }^{6}$ Sarnia Community Care Physiotherapy, Sarnia, Ontario N7T 7W5, Canada and ${ }^{7}$ Université de Sherbrooke, Sherbrooke, Quebec J1K 2R1, Canada

Email: Joy C MacDermid* - jmacderm@uwo.ca; Myriam Ghobrial - myriam_ghobrial@ hotmail.com;

Karine Badra Quirion - sea_kwinz@hotmail.com; Mélanie St-Amour - mstamour23@cogeco.ca; Tanya Tsui - t_tsui@hotmail.com;

Dave Humphreys - dchumphreys@rogers.com; John McCluskie - mccluski@hhsc.ca; Eddy Shewayhat - eshewayhat@hotmail.com;

Vickie Galea - galeav@mcmaster.ca

* Corresponding author
\end{abstract}

Published: 17 May 2007

BMC Musculoskeletal Disorders 2007, 8:42 doi:10.1 I86/I47|-2474-8-42

This article is available from: http://www.biomedcentral.com/I47I-2474/8/42

(c) 2007 MacDermid et al; licensee BioMed Central Ltd.

This is an Open Access article distributed under the terms of the Creative Commons Attribution License (http://creativecommons.org/licenses/by/2.0), which permits unrestricted use, distribution, and reproduction in any medium, provided the original work is properly cited.
Received: 30 June 2006

Accepted: 17 May 2007

\begin{abstract}
Background: There is a lack of standardized tests that assess functional performance for sustained upper extremity activity. This study describes development of a new test for measuring functional performance of the upper extremity and neck and assesses reliability and concurrent validity in patients with shoulder pathology.
\end{abstract}

Methods: A series of developmental tests were conducted to develop a protocol for assessing upper extremity tasks that required multi-level movement and sustained elevation. Kinematics of movement were investigated to inform subtask structure. Tasks and test composition were refined to fit clinical applicability criteria and pilot tested on 5 patients awaiting surgery for shoulder impingement and age-sex matched controls. Test-retest reliability was assessed on 10 subjects. Then a cohort of patients with mild to moderate $(n=17)$ shoulder pathology and 19 controls $(17$ were age-sex matched to patients) were tested to further validate the Functional Impairment TestHand, and Neck/Shoulder/Arm (FIT-HaNSA) by comparing it to self-reported function and measured strength. The FIT-HaNSA, DASH and SPADI were tested on a single occasion. Impairments in isometric strength were measured using hand-held dynamometry. Discriminative validity was determined by comparing scores to those of age-sex matched controls $(n=34)$, using ANOVA. Pearson correlations between outcome measures $(n=4 I)$ were examined to establish criterion and convergent validity.

Results: A test protocol based on three five-minute subtasks, each either comprised of moving objects to waist-height shelves, eye-level shelves, or sustained manipulation of overhead nuts/bolts, was developed. Test scores for the latter 2 subtasks (or total scores) were different between controls as compared to either surgical-list patients with shoulder impingement or a variety of 
milder shoulder pathologies $(p<0.0 I)$. Test I correlated the highest with the DASH $(r=-0.83)$, whereas Test 2 correlated highest with the SPADI $(r=-0.76)$.

Conclusion: Initial data suggest the FIT-HaNSA provides valid assessment of impaired functional performance in patients with shoulder pathology. It discriminates between patients and controls, is related to self-reported function, and yet provides distinct information. Longitudinal testing is warranted.

\section{Background}

Given that shoulder pathology has a substantial impact on quality of life [1-5] that is lasting [6,7], and only 59\% of shoulder complaints resolve within 12 months [8], reliable and valid methods of determining risk, severity, and outcome for these disorders are imperative.

Strength and stability must co-exist to insure optimal shoulder function. Given the key role of muscles in establishing shoulder stability, mobility, and function, it is not surprising that the strength of specific muscle groups is typically viewed as a key outcome measure when evaluating shoulder conditions. Quantitative measures of isometric [4,9-12] and isokinetic [4,11-23] strength have been described to dictate the functionality of muscles in different muscle actions. The importance of muscle strength has been confirmed in that shoulder strength has been shown to be related to general health status in persons with shoulder pathology [4]. Range of motion (ROM) [24] is also frequently reported in shoulder studies, although its relationship to function has been less clearly defined [25]. Shoulder functionality requires coordinated, sustained muscle activity that both maintains sufficient proximal control and also allows a wide arc of pain-free movement for completion of tasks of daily life. Thus, it is not unexpected that isolated physical impairments like muscle strength or ROM deficits have demonstrated small to moderate correlation to function. However, this suggests that better understanding of function requires specific functional tests.

One approach to "functional outcomes" has been the use of patient-self report. Pain is a primary symptom in most shoulder disorders. In fact, shoulder pain has been a primary focus of systematic reviews looking at diagnosis [26], prognosis [6], and treatment effectiveness [27,28]. These reviews have emphasized the need for validated outcome measures to improve the validity of clinical trials addressing shoulder pain [27]. A number of self-report measures have been designed to assess shoulder pain and disability from the patient's perspective. These include disease-specific quality-of-life measures [29,30], jointspecific pain/disability measures [31], and regional measures [32,33]. Self-report measures have the advantage of being patient-centered and relatively easy and inexpensive to apply. They provide an important perspective on patient status. However, studies conducted in different upper extremity clinical populations [34-37], and specifically, shoulder problems $[4,38,39]$ agree that the relationship between self-reported disability and actual physical impairment or functional performance is moderate [40]; thus, they cannot be used as surrogates. This suggests the need for a valid structured test of functional performance of the upper extremity that incorporates shoulder and neck.

While a variety of hand function or dexterity tests have been described [41-46], most tasks involve minimal shoulder movement, nor were they developed or validated to assess patients across a spectrum of shoulder conditions. An exception is the test developed by Hughes et al. [47], the Simple Shoulder Endurance Test. Unfortunately, this test assesses only one task and demonstrated limited reliability. There are also reports of "endurance" or "fatigue" protocols that can be used on isokinetic dynamometers [48-51], although most last less than 2 minutes, suggesting they do not assess the physiologic pathways used in most sustained work or functional activity.

Therefore, our aim was to (i) develop a test protocol that requires coordinated movement and positioning of the upper extremity and neck across different tasks that simulate elements of functional activity, and (ii) test its validity and reliability in a first phase diagnostic study.

\section{Methods}

\section{Study overview}

1. Phase 1 - Protocol Development

2. Phase 2 - Psychometric Evaluation: (Preliminary) Reliability Testing

3. Phase 3 - Psychometric Evaluation: Validation of FITHaNSA in patients with mild-moderate shoulder pathology

\section{Protocol development}

The test was developed to address tasks of functional relevance that challenged the upper quarter, including shoulder/neck, with content relevance determined by both clinical experience and kinematic analyses. Preliminary 
task definition that described the nature of the tasks was completed in the Human Movement Laboratory at McMaster University. The kinematics of the upper extremity during a reach and grasp task were evaluated in a series of studies evaluating motor control of the upper extremity and neck (described below). Literature describing the kinematics of shoulder function tests [47], hand dexterity tests $[41,42,45,46,52-54]$, the first author's experience in test development, and problems reported by patients with shoulder conditions were also used to develop the preliminary protocol. The following criteria for relevance and clinical utility were established as necessary components during test development:

1. have a variety of subtasks that included gross motor and sustained movements of the upper extremity and neck including shoulder/neck/elbow.

2. should be relevant to a wide spectrum of disorders by having subtasks that would be completed by subjects with severe pathology as well as those with mild pathology.

3. should be completed using readily available commercial equipment or be easily constructed from routine materials.

\section{4. should be easily scored.}

5. should assess endurance to sustained "functional" activity in a standardized format, i.e., > 5 minutes of muscle activity, but be reasonable to perform in a clinical environment, i.e., < 20 minutes using standardized methods.

Task definition was conducted on 12 volunteers with no neck, shoulder, arm, nor hand pathology. The age range for this set of subjects was 19-21 years since all were undergraduate university students. Segmental 3-dimensional kinematic behaviour was acquired using a magnetically-based motion tracker (Polhemus Systems, Skills Technology). We acquired the 3-dimensional position and orientation data through electromagnetic sensors placed on the neck (upper trunk), upper arm, forearm, and hand segments to represent the shoulder, elbow, and wrist joints. The joint angular displacement time series were most informative to the "challenge" required for patients with various neck, shoulder, and arm pathologies to perform. As an example, the ranges of motion required for the "Repetitive High Height" task were at least $120^{\circ}$ of shoulder flexion, $25^{\circ}$ of shoulder abduction, and at least $30^{\circ}$ of external rotation (See Figure 1 ). These ranges had to be sustained throughout the testing period ( 3 minutes) at a metronome speed of 60 beats per minute.

Potential "pilot" tasks were reviewed by the first author and reconfigured to fit with the criteria for relevance and
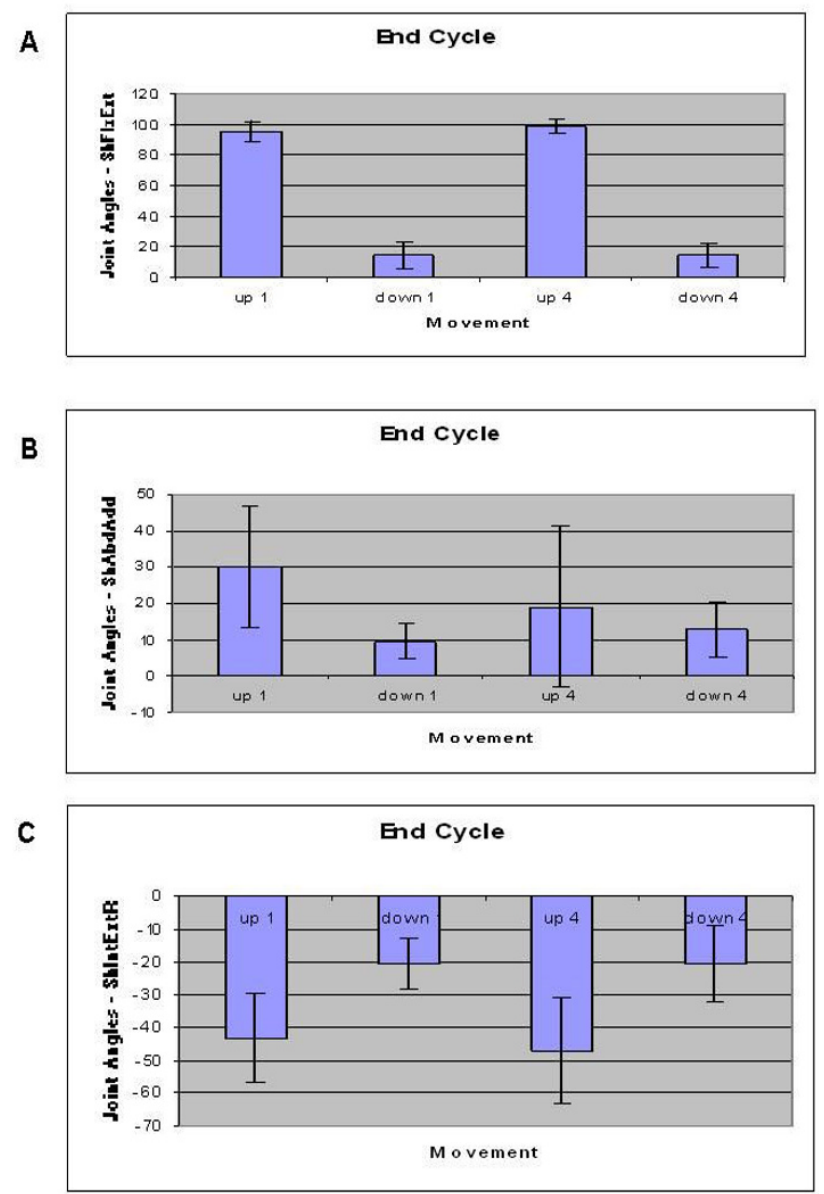

Figure I

Shoulder ROM during repetitive high height task. Values acquired from development studies. A - Shoulder Flexion/ Extension; B - Shoulder Abduction/Adduction; C - Shoulder Internal/External Rotation.

clinical utility. During the next phase of test development, knowledgeable physiotherapists and physiotherapy students reviewed potential test components. Issues on spectrum of testing, test components, test procedures, scoring, and the potential influence of different pathologies were discussed to insure that the test components would be feasible and useful for clinical assessment of patients. Iterative test development sessions were conducted to refine this "alpha protocol". Minor changes were made to increase the difficulty of the tasks and enhance their clinical application.

\section{The test protocol}

The final functional assessment protocol is called the Functional Impairment Test-Hand and Neck/Shoulder/ Arm (FIT-HaNSA) and consists of a battery of three tasks that simulate daily activities of lifting and sustained over- 
head work in the household or workplace. FIT-HaNSA was designed to test the endurance of the shoulder, with the expectation that the difficulty of the sustained tasks would distinguish between individuals with varying degrees of shoulder function with the relative task difficulty depending on the nature of the underlying shoulder pathology. The JobSim (JTech Medical, Salt Lake City, USA) was used for all tests (Figure 2); however, the test could also be reproduced with commercial shelving/hardware or custom-made materials. In fact, a wooden version is currently in use in our Human Movement Laboratory (Figure 3) (see additional file 1: The FIT-HaNSA Protocol for a detailed description) for studies which require the use of electromagnetic devices.

The test consists of 3 subtasks, the first being the simplest to perform for the majority of individuals. A test manual

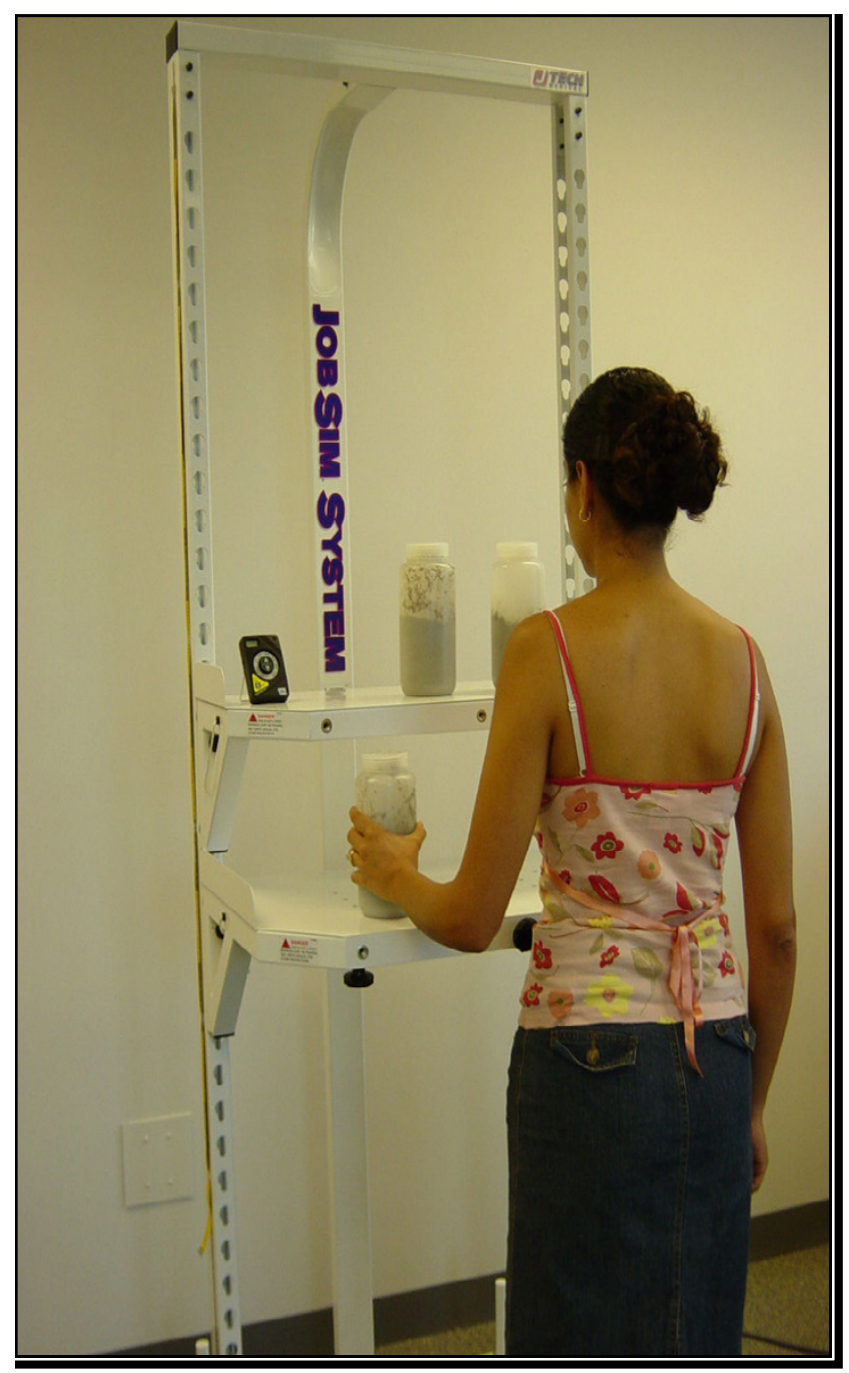

Figure 2

Task I, "Waist-Up". is provided as a supplementary file (See additional file 1: The FIT-HaNSA Protocol). Each task can be continued for up to 5 minutes, but is terminated based on the following stopping rules:

1. The subject stops or states it is too painful to continue.

2. The subject is severely off pacing (provided by a metronome) to the extent that they are unable to complete one repetition of the movement within 2 beats of the metronome (for 5 successive repetitions).

3. The subject substitutes using trunk/whole body movement and cannot correct with feedback for 5 successive repetitions of the task.

4. The examiner believes the subject is at risk of injury or adverse events if the test were to continue.

In the first task ("waist-up"), a shelf was placed at waist level and a second shelf was placed $25 \mathrm{~cm}$ above it; three $1-\mathrm{kg}$ containers were placed $10 \mathrm{~cm}$ apart on the lower shelf (Figure 2). Using the affected arm, the patient would

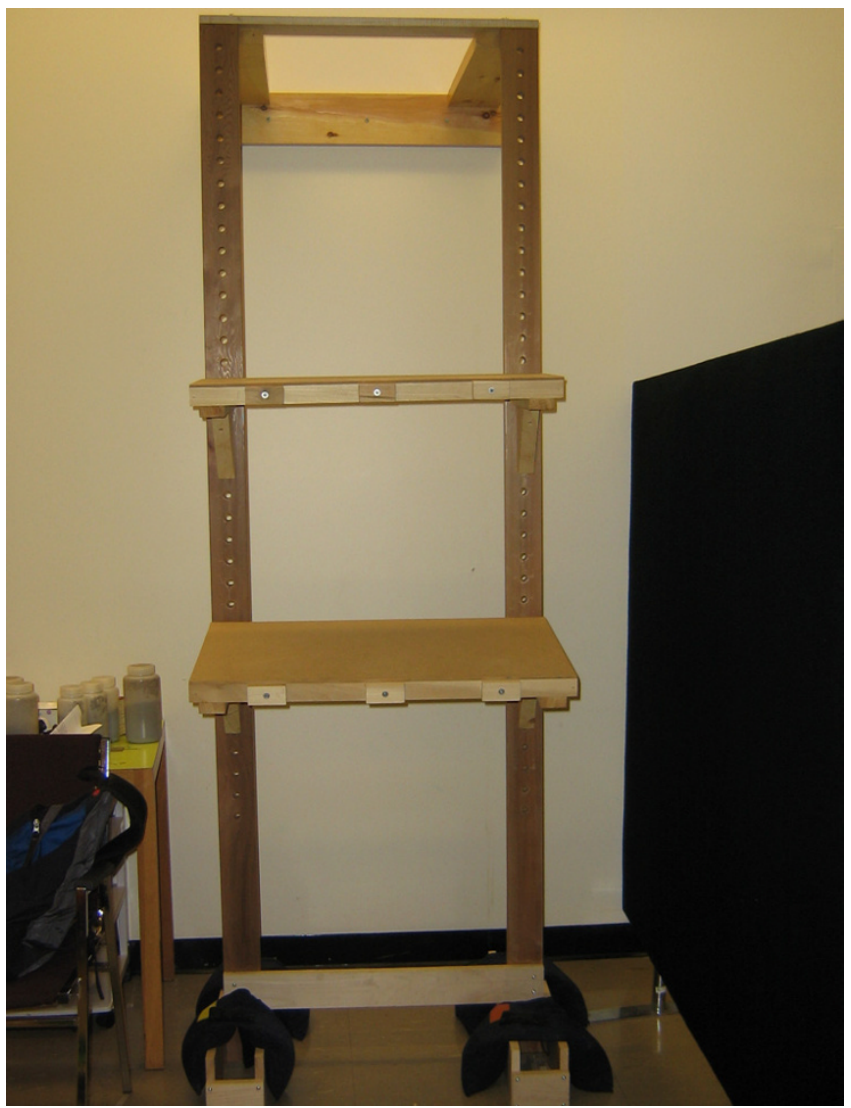

Figure 3

Wooden Version of the FIT-HaNSA. 
lift the 3 containers, one at a time, from one shelf to the other at a speed of 60 beats per minute, controlled by a metronome. The subjects and controls were instructed to do the test until 5 minutes have elapsed or they feel unable to continue. The time to complete the task was measured by a stopwatch.

In the second task ("eye-down"), the shelves were adjusted so that one shelf was placed at the subject's eye level and the second shelf was placed $25 \mathrm{~cm}$ below it (Figure 4). The patients were again instructed to use their affected arm to lift the three containers between the shelves at a speed of 60 beats per minute. The same stopping protocol was used for task 2.

In task 3 ("overhead work"), a shelf was placed at the subject's eye level with an attachable plate, perpendicular to

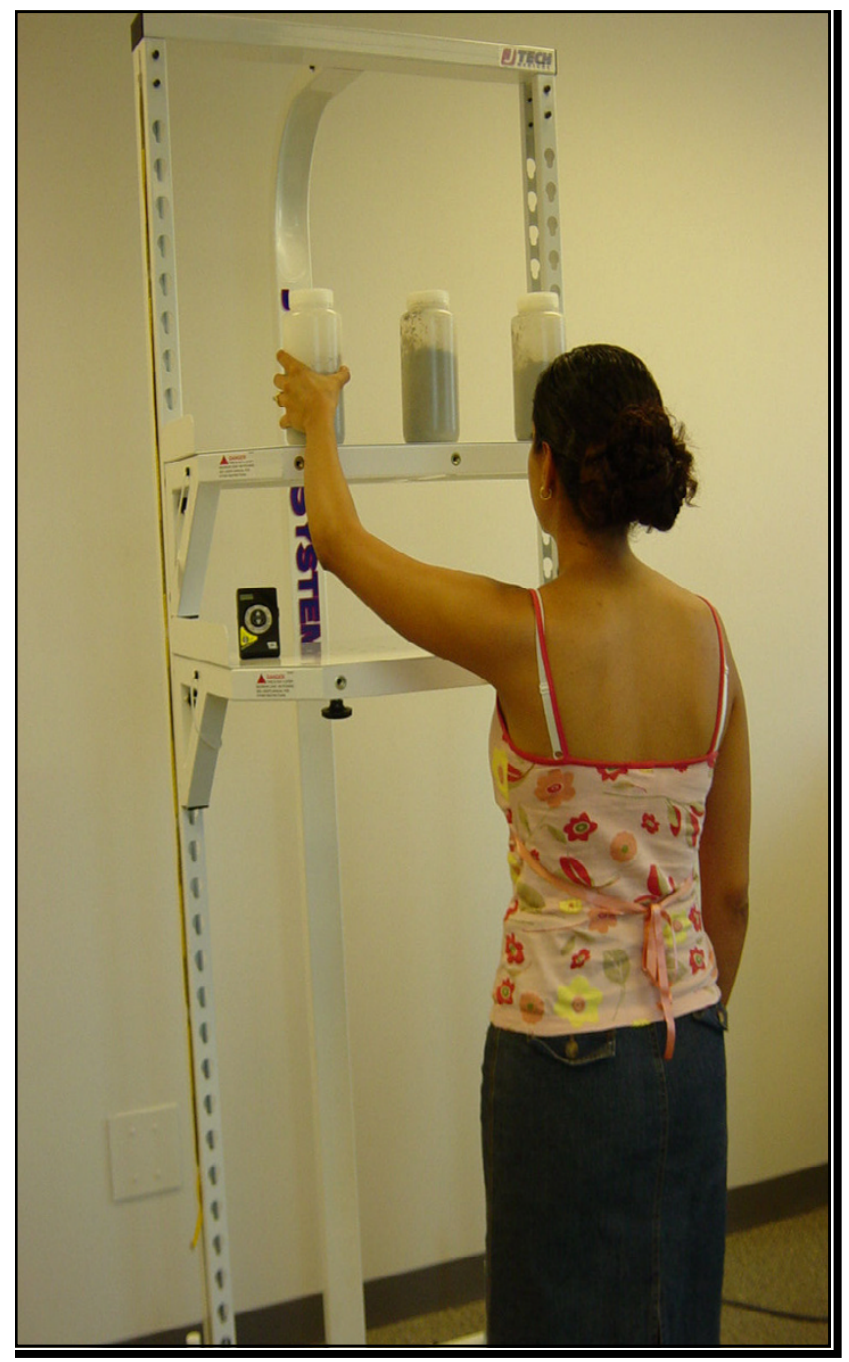

Figure 4

Task 2, "Eye-Down". the shelf, projecting out toward the subject (Figure 5). Patients were instructed to use their affected arm to repeatedly screw and unscrew bolts in a pattern (the bolt in notch 1 (top) moves down to notch 2 (middle); the bolt in notch 3 (bottom) moves up to notch 1; the bolt in notch 2 moves down to notch 3 ) into the plate. The same stopping protocol was used for task 3 . Arms were not dropped during testing.

There was an approximately 30-second rest between the tests as the shelves were adjusted for the different tasks.

\section{Psychometric objectives}

1. To develop a test protocol that could be completed by most subjects without shoulder pathology, but sensitive to detecting functional impairment across a spectrum of severity (mild to severe).

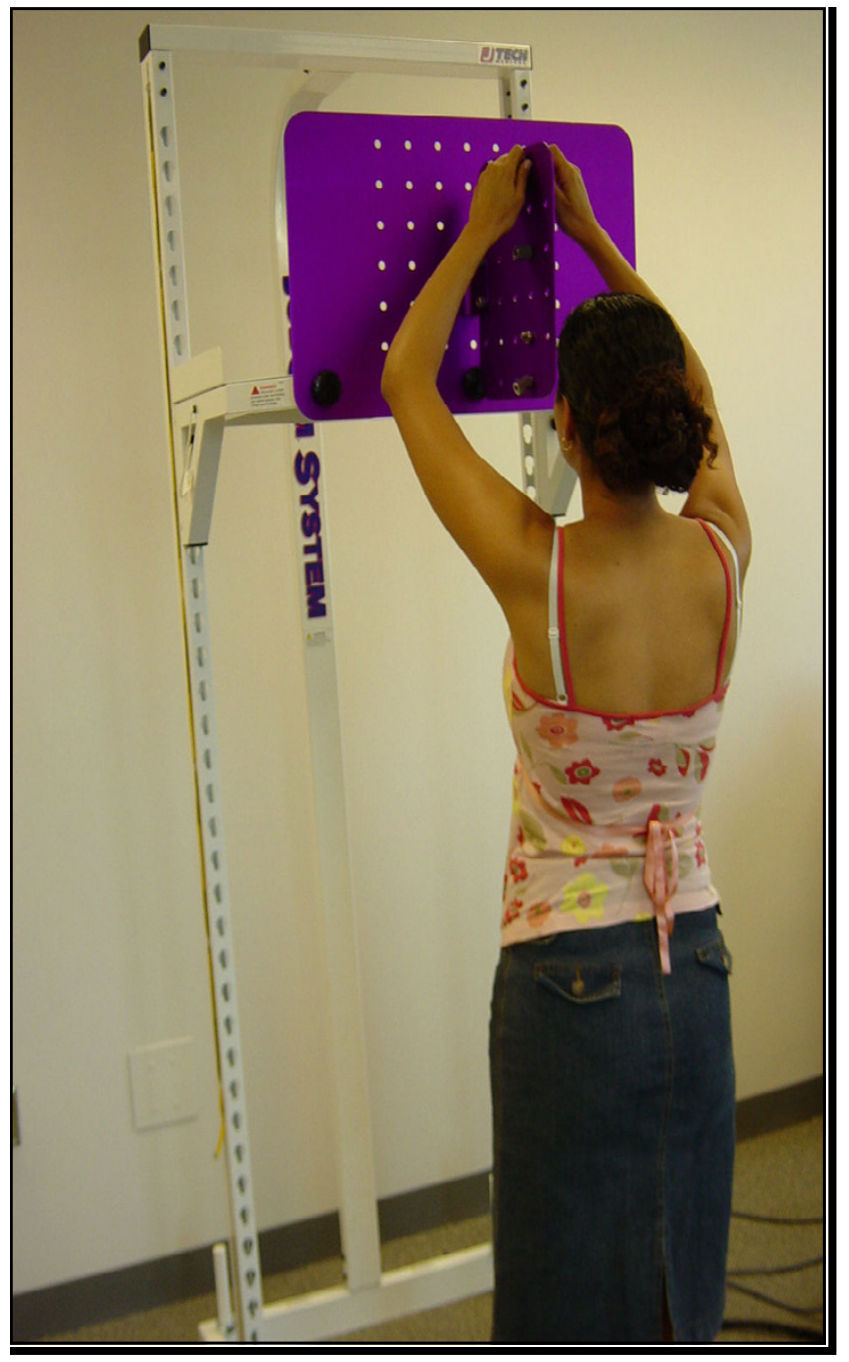

Figure 5

Task 3, "Overhead Work". 
2. To describe the central tendency and variability of this test in subjects with shoulder pathology as compared to age-sex matched controls.

\section{To provide preliminary estimates of reliability.}

4. To determine the concurrent convergent validity by comparing the new test to indicators of impairment in patients with shoulder disorders-including self-reported function and isometric muscle strength.

5. To determine the construct validity of the new test by assessing whether the following constructed hypotheses were supported:

a. Subjects with mild-moderate impingement would be different from controls.

b. Subjects with impingement would have minimal difficulty with a low level reach task with light weight, but more difficulty with tasks in impinged positions.

\section{Participants}

Group I - Early validation in high severity subgroup

Subjects $(n=5)$ were recruited from the surgical wait-list of a single orthopedic surgeon. Patients with a diagnosis of shoulder impingement unresponsive to conservative management but without complicating co-morbidity were tested on a single occasion. Subjects were identified as having shoulder impingement on the basis of a physical examination by a shoulder surgeon which was further confirmed on the test occasion by a positive Hawkins test [55-57]. Control subjects were sex- and age-matched $( \pm 5$ years). The age range of subjects was 25-76. The Hamilton Health Sciences Research Ethics Board approved the protocol.

\section{Group 2 - Reliability testing}

A subgroup of patient and controls $(n=10)$ who agreed to duplicate testing were evaluated on two occasions; Mean age $=39 \pm 14 ; 2 \mathrm{M}, 8 \mathrm{~F}$ (3 patients, 7 controls).

\section{Group 3 - Validation in mild-moderate shoulder pathology}

Thirty-six subjects were recruited in total; patients with shoulder pain $(n=17)$ were recruited from the sports medicine/staff physiotherapy clinic at McMaster University (see Table 1). The patients' shoulder pathologies included: bicipital tendonitis, bursitis, chronic subluxation, capsulitis, impingement, instability, and surgical repair of the labrum. Shoulder patients were not excluded if they also reported hand, elbow, or neck pathology. The control group consisted of nineteen subjects with no shoulder pathology as indicated by self-report (direct question and SPADI scores); 17 were sex- and agematched $( \pm 5$ years) to tested patients. The mean age of
Table I: Demographics of Validation Study Participants

\begin{tabular}{lcc}
\hline & Controls (n = I9) & Subjects (n = I7) \\
\hline Age (yrs) & $32($ I2) & $32(12)$ \\
Sex & II females, 8 males & 9 females, 8 males \\
Flexion (lbs of F) & 34.1 I (I4.67) & $30.46(14.30)$ \\
Abduction (lbs of F) & $33.75(14.20)$ & $30.55(13.41)$ \\
DASH & $1.10(1.57)$ & $10.25(12.57)$ \\
SPADI & $1.26(2.40)$ & $16.41(18.60)$ \\
Test I (secs) & $300(0)$ & $300(0)$ \\
Test 2 (secs) & $290(25)$ & $246(74)$ \\
Test 3 (secs) & $300(0)$ & $275(51)$ \\
Ave. total time (secs) & $297(8)$ & $274(34)$ \\
\hline
\end{tabular}

Mean (Standard Deviation)

the experimental and control subjects was 32 years (range = 20-62 years). The McMaster University Ethics Board approved the study and informed consent was obtained from all subjects prior to testing.

\section{Comparative measures}

All subjects completed the Disabilities of the Arm, Shoulder, Hand (DASH) $[33,58]$, and the Shoulder Pain and Disability Index (SPADI) $[31,59,60]$. In part 3 of the study, isometric shoulder abduction and flexion strength for the test shoulder were measured using the Lafayette Manual Muscle Tester (Lafayette Instrument Company, Lafayette, USA). Isometric strength testing was performed in a stable seated position with the arm in neutral (3 repetitions averaged).

\section{Analyses}

During the developmental work (phase 1), the tests were modified so that the instructions were standardized, the break between subtasks (to further emphasize endurance) was decreased, and the motions performed were clarified. In phase 2, Intra-class Correlation Coefficients $(2,1)$ [61] were used to describe test-retest reliability. For all data subgroups, scale scores and variability, descriptive statistics of the task, and test performance were computed. For phase 3 validity analyses, differences were determined between subgroups by using ANOVA. Pearson correlations were used to compare strength and self-reported function measures to FIT-HaNSA scores. All statistical analyses were conducted in SPSS 14.0.

\section{Results}

\section{Early validation (severe pathology)}

Data provided by group 1 provided preliminary data (wide variation/small sample size) that compared subjects with severe shoulder impingement to age-matched controls with no shoulder pathologies. Patient stopped the subtests for a combination of discomfort and fatigue. The mean times for tasks 1, 2, and 3 for those with shoulder impingement were 179 s, 117 s, and 151 s, respec- 
tively. Controls completed all subtasks except for Task 2 in 1 control, providing means of $300 \mathrm{~s}, 286 \mathrm{~s}$, and $300 \mathrm{~s}$, respectively, for those with no shoulder pathologies. Despite the small sample, the mean times for tasks 1, 2, 3 for subjects with shoulder impingement were significantly shorter than the age-sex matched control group ( $\mathrm{p}=$ 0.041). The percentage of task completion (mean time spent on all three tests/300 s) for subjects $1,2,3,4$, and 5 were $33 \%, 12 \%, 22 \%, 86 \%$, and $95 \%$, respectively, when using $300 \mathrm{~s}$ as the referent standard. The Pearson correlation coefficients between FIT-HaNSA and the SPADI or DASH were -0.85 and -0.94 , respectively.

\section{Reliability}

ICCs $(2,1)$ (95\% confidence intervals) for test-retest reliability of scores for Group 2 were:

\section{Test 1: 0.97 (0.84-0.99)}

2. Test 2: $0.98(0.94-1.00)$

3. Task 3: $0.96(0.85-0.99)$

\section{3-Task Mean Score: 0.98 (0.90-0.99)}

\section{Validation in mild-moderate pathology}

In group 3, the patients' mean times with 95\% confidence intervals in brackets for tasks 1,2 , and 3 were $300 \mathrm{~s}, 246 \mathrm{~s}$ (208-284), and $275 \mathrm{~s}$ (249-300), respectively, whereas the controls' mean times were $300 \mathrm{~s}, 290 \mathrm{~s}(278-300)$, and $300 \mathrm{~s}$, respectively (Table 1). The patients' total mean

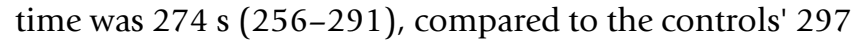
s (293-300). There was no significant difference between patients and controls for isometric strength. Significant differences were found between the groups for the DASH $(p=0.005)$, the SPADI $(p=0.002)$, and the average total time on FIT-HaNSA $(\mathrm{p}=0.008)$. Test 2 demonstrated the largest difference between patients and controls $(\mathrm{p}=$ 0.021 ). Test 3 was also statistically different between the groups $(\mathrm{p}=0.044)$ (Figure 6$)$. Test 1 correlated the highest with the DASH (0.01 level, $\mathrm{r}=-0.83)$, whereas Test 2 correlated highest with SPADI (0.01 level, $r=-0.76)$ (Table 2 ). The correlations between isometric strength scores and the DASH or SPADI were non-significant.

\section{Discussion}

This study describes the development of a new functional performance test for shoulder pain patients and provides preliminary results for its validity and reliability. Discriminative validity and construct/criterion validity have been supported in two groups of patients. In a small group of more severely affected patients with uncomplicated impingement awaiting surgery, the differences in scores were profound and statistically significant despite a very small sample size. In patients with milder conditions who

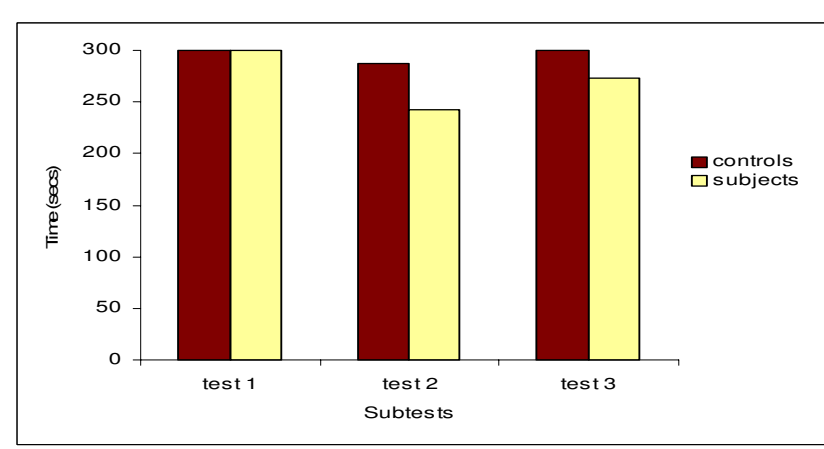

Figure 6

Comparison of Mean Group Times. This figure shows FITHaNSA times for shoulder patients versus controls. Significant differences were observed for Task 2 and Task 3. All subjects performed 5 full minutes of Task $I$.

were involved in active rehabilitation, the differences were less extreme but also statistically significant. It is noteworthy that isometric strength scores were not affected in this same group, suggesting that the FIT-HaNSA was better able to detect the physical impairments associated with these milder pathologies (i.e., was more discriminative). Preliminary reliability testing of a small mixed group was encouraging with all reliability coefficients exceeding 0.95 .

Our test was designed to be assessed in a spectrum of shoulder patients. Task 1 is the first and easiest to perform. We anticipated that subjects with severe shoulder pathology like total shoulder arthoplasty, severe osteoarthritis, or with proximal humeral fractures might have difficulty with this task. However, we did not test that assumption. Clearly, this task contributes to the overall endurance load placed the shoulder, but was included in the protocol to insure that floor effects were not a problem. It will be important to determine whether this sub-

Table 2: Pearson correlations between the FIT-HaNSA tasks and strength or self-report scores

\begin{tabular}{lccc}
\hline & Task I & Task 2 & Task 3 \\
\hline Flexion strength & -- & $\begin{array}{c}0.29 \\
(\mathrm{n}=36) \\
0.27\end{array}$ & $\begin{array}{c}0.15 \\
(\mathrm{n}=36) \\
0.12\end{array}$ \\
Abduction strength & -- & $\begin{array}{c}(\mathrm{n}=36) \\
(\mathrm{n}=36)\end{array}$ \\
DASH & $-0.83^{* *}$ & $\begin{array}{c}-0.82^{* *} \\
(\mathrm{n}=4 \mathrm{I})\end{array}$ & $\begin{array}{c}-0.73^{* *} \\
(\mathrm{n}=4 \mathrm{I})\end{array}$ \\
SPADI & $(\mathrm{n}=4 \mathrm{I})$ & $\begin{array}{c}-0.76^{* *} \\
-0.67^{* *}\end{array}$ \\
& $-0.74^{* *}$ & $(\mathrm{n}=4 \mathrm{I})$ & $(\mathrm{n}=4 \mathrm{I})$ \\
\hline
\end{tabular}

** Correlation is significant at the 0.01 level (2-tailed).

Note: The correlations between task I and strength could not be computed because all subjects completed the 300 -seconds task. The correlations between the tasks and self-report scores included the 5 patients from the development phase (part I). 
task detects a spectrum of capability in patients with more severe shoulder conditions.

We recognize limitations in specific examination tests and in the process of making definitive diagnoses in shoulder disorders, particularly for "shoulder impingement" which is really a symptom not a diagnosis. Nevertheless, "impingement" is one of the most common problems affecting the shoulder $[62,63]$ and its presence in our subjects was confirmed by expert orthopedic surgeons and physiotherapists. Task 2, which involves repetitive movement into a relatively impinged position during a grasp and place activity, was anticipated to be challenging for these patients. In our developmental work with patients who have severe impingement, this was very evident as task 2 was grossly limited and more limited than other tasks. Our second validation group included a spectrum of patients including a number with impingement and again, Task 2 was the most challenging. Due to the nature of the test protocol used in Phase 2, we had longer breaks between subtests and this may also have contributed to better performance on subsequent tasks. We suggest that the appropriate rest between subtasks should be the time required to adjust shelves (less than 30 seconds).

We had anticipated that Task 3, where sustained overhead work is performed, might be most challenging for patients with shoulder/neck pain, particularly if associated with radiculopathy or for other conditions involving neural structures such as thoracic outlet syndrome. Again, these subgroups were not tested so this assumption is also not yet validated. However, the range of tasks included in FITHaNSA is consistent with a range of functional activities that would be affected in shoulder disorders.

There are a number of variations between FIT-HaNSA and the other shoulder functional test reported in the literature. The "Simple Shoulder Endurance Test" reported by Hughes [47] consists of a single activity (screwing bolts) motion that required shoulder stability at $45^{\circ}$ of forward flexion, but not movement of the shoulder. The task was performed with increasing weight every 2 minutes and the average amount of time to test termination was $413 \mathrm{sec}-$ onds. This is similar to our 300-second subtask time, but there are advantages to our staged 3-level subtask approach as compared to the single task protocol described above. One advantage of FIT-HaNSA is that it tests multiple functions of the shoulder and neck, in particular, assessing both stabilization and movement. We used a standing position as the sitting and belted position does not represent the way most functional tasks would be performed. A further advantage is that functional endurance is assessed over a longer period of time which may be more reflective of actual functional performance.
We were encouraged by a number of findings. The discriminative validity of FIT-HaNSA was greater than demonstrated by isometric strength testing with a hand-held dynamometer. This suggests that the test is providing useful information about the physical status in mild to moderate shoulder disorders. Reliability scores, while based on a small and broadly-based sample, were excellent. We anticipate that reliability coefficients may be lower when the test is performed in more homogeneous populations, as a more variable population favors achieving high ICCs. Nevertheless, favourable preliminary reliability evaluation indicates that these investigations of test behaviour in different subpopulations are indicated.

While the reliability, discriminative validity, and score ranges demonstrated in this study may imply an ability for the test to evaluate change over time, our study was crosssectional and, thus, we are unable to make such conclusions. We acknowledge these results are preliminary and purposively wish to share the protocol at this early stage to allow others to implement it and potentially to participate in independent validation. While the numbers reported in this study are small, 2 years of development and piloting was conducted to devise and evaluate a protocol that is ready for widespread clinical field testing. It is important that while we proceed with additional validation, independent authors have the opportunity to do as well. This should include different types of upper extremity/neck pathology, across different age/sex subgroups and test environments. Substantial gaps in knowledge about the psychometrics of this test remain, notably the longitudinal validity, including responsiveness. This study reports the iterative development process and early positive psychometrics properties of a new functional performance test.

\section{Conclusion}

This study provides preliminary support for the validity of the FIT-HaNSA in clinical evaluation of shoulder disability in that it is tolerable for most people without pathology, completed in 20 minutes, has acceptable test-retest reliability, and discriminates between controls and patients in both severe and mild to moderate shoulder pathology. It provides different information than selfreported function and, thus, provides an alternative means to assess functional status or progression of functional ability over time. Future studies should focus on evaluating its responsiveness in longitudinal studies and its psychometric properties across different upper extremity pathologies.

\section{Competing interests}

The author(s) declare that they have no competing interests. 


\section{Authors' contributions}

VG has conducted work on kinematics of the upper limb and discussed this with JM when FIT-HaNSA was being developed. JM developed the preliminary clinical test protocol and worked with 2 research groups to refine the test protocol and validate it. The first group (DH, JM, ES) helped with test protocol development and tested patients awaiting surgery for surgery for impingement repair, and the second study group (MG, KBQ, MS, TT) evaluated FITHaNSA in patients with a spectrum of milder shoulder pathologies. JM conducted statistical analyses and drafted the manuscript. All authors approved the final study protocol, contributed to interpretation of the study results, and participated in revisions of the manuscript. All authors read and approved the final manuscript.

\section{Additional material}

\section{Additional File 1 \\ The FIT-HaNSA Protocol \\ Click here for file \\ [http://www.biomedcentral.com/content/supplementary/1471- \\ 2474-8-42-S1.doc]}

\section{Acknowledgements}

We are grateful for financial support received from the Canada Foundation for Innovation (CFI) that funded the equipment and clinical lab where this test was developed. Joy MacDermid holds a New Investigator Award from the Canadian Institutes for Health Research. Thanks to Margaret Lomotan for testing subjects and assistance with manuscript preparation. Thanks to James Bromley for building and giving us permission to use a picture of the wooden shelving unit. Thanks to Prajyot Kumta for assisting with collection of test-retest data.

\section{References}

I. Hollinshead RM, Mohtadi NG, Vande Guchte RA, Wadey VM: Two 6-year follow-up studies of large and massive rotator cuff tears: comparison of outcome measures. J Shoulder Elbow Surg 2000, 9:373-381.

2. Razmjou H, Bean A, van $O$ V, Mac Dermid JC, Holtby R: Cross-sectional and longitudinal construct validity of two rotator cuff disease-specific outcome measures. BMC Musculoskelet Disord 2006, 7:26.

3. Razmjou H, Holtby R, Myhr T: Gender differences in quality of life and extent of rotator cuff pathology. Arthroscopy 2006, 22:57-62.

4. MacDermid JC, Ramos J, Drosdowech D, Faber K, Patterson S: The impact of rotator cuff pathology on isometric and isokinetic strength, function, and quality of life. J Shoulder Elbow Surg 2004, 13:593-598.

5. Matsen FA III, Antoniou J, Rozencwaig R, Campbell B, Smith KL: Correlates with comfort and function after total shoulder arthroplasty for degenerative joint disease. J Shoulder Elbow Surg 2000, 9:465-469.

6. Kuijpers T, van der Windt DA, van der Heijden GJ, Bouter LM: Systematic review of prognostic cohort studies on shoulder disorders. Pain 2004, 109:420-431.

7. Largacha M, Parsons IM, Campbell B, Titelman RM, Smith KL, Matsen F III: Deficits in shoulder function and general health associated with sixteen common shoulder diagnoses: a study of 2674 patients. J Shoulder Elbow Surg 2006, I 5:30-39.
8. van der Windt DA, Koes BW, Boeke AJ, Deville W, De Jong BA, Bouter LM: Shoulder disorders in general practice: prognostic indicators of outcome. Br J Gen Pract 1996, 46:519-523.

9. Balogun JA, Powell R, Trullender B, Olson S, Balogun AO: Intra- and inter-tester reliability of the NICHOLAS hand-held dynamometer during evaluation of upper extremity isometric muscle strength. European Journal of Physical Medicine and Rehabilitation 1998 May; 8:48-53.

10. Desrosiers J, Rochette A, Payette H, Gregoire L, Boutier V, Lazowski $D$ : Upper extremity isometric strength measurement using the belt-resisted method: reliability study with healthy elderly people. Canadian Journal of Rehabilitation, I I(3):I49-55, 1998 Spring (I5 ref) 1998, I I: |49- I 55.

II. Kuhlman JR, lannotti JP, Kelly MJ, Riegler FX, Gavaert ML, Ergin TM: Isokinetic and isometric measurement of strength of external rotation and abduction of the shoulder. J Bone Joint Surg I992, 74A: | 320-I333.

12. Leggin BG, Neuman RM, lannotti JP, Williams GR, Thompson EC: Intrarater and interrater reliability of three isometric dynamometers in assessing shoulder strength. J Shoulder Elbow Surg 1996, 5:18-24.

13. Barton MA, Wallace WA, Robinson N: Isokinetic strength of the shoulder after repair of a torn rotator cuff. J Bone Joint Surg Am 1988, 70: 1270-127|.

14. Brown LP, Niehues SL, Harrah A, Yavorsky P, Hirshman HP: Upper extremity range of motion and isokinetic strength of the internal and external shoulder rotators in major league baseball players. Am J Sports Med 1988, I 6:577-585.

15. Cahalan TD, Johnson ME, Chao EY: Shoulder strength analysis using the Cybex II isokinetic dynamometer. Clin Orthop 1991:249-257.

16. Dauty M, Delbrouck C, Huguet D, Rousseau B, Potiron-Josse M, Dubois C: Reproducibility of concentric and eccentric isokinetic strength of the shoulder rotators in normal subjects $\mathbf{4 0}$ to 55 years old. Isokinetics and Exercise Science 2003; I I:95-100.

17. Greenfield B, Johanson M: Isokinetic and isometric measurement of strength of external rotation and abduction of the shoulder [letter]. J Bone Joint Surg Am 1993, 75: I 254-I 254.

18. Greenfield BH, Donatelli R, Wooden MJ, Wilkes J: Isokinetic evaluation of shoulder rotational strength between the plane of scapula and the frontal plane. Am J Sports Med I990, I 8: I 24-I 28.

19. Hartsell HD, Forwell L: Postoperative eccentric and concentric isokinetic strength for the shoulder rotators in the scapular and neutral planes. J Orthop Sports Phys Ther 1997, 25: 19-25.

20. Leroux JL, Codine P, Thomas E, Pocholle M, Mailhe D, Blotman F: Isokinetic evaluation of rotational strength in normal shoulders and shoulders with impingement syndrome. Clin Orthop 1994:108-II5.

21. Magnusson SP, Gleim GW, Nicholas JA: Subject variability of shoulder abduction strength testing. Am J Sports Med 1990, 1 8:349-353.

22. Mayer F, Baur H, Hirschmuller A, Heitkamp HC, Horstmann T, Dickhuth $\mathrm{HH}$ : The quantification of reciprocal shoulder strength relation in various working modes at different movement velocities. Isokinetics and Exercise Science 200 I; 9:73-77.

23. van Meeteren J, Roebroek ME, Stam HJ: Test-retest reliability in isokinetic muscle strength measurements of the shoulder. J Rehabil Med 2002, 34:91-95.

24. MacDermid JC, Chesworth BM, Patterson S, Roth JH: Intratester and intertester reliability of goniometric measurement of passive lateral shoulder rotation. J Hand Ther 1999, I 2:187-192.

25. MacDermid JC, Chesworth BM, Patterson S, Roth JH: Validity of pain and motion indicators recorded on a movement diagram of shoulder lateral rotation. Australian Journal of Physiotherapy 1999, 45:269-277.

26. Dinnes J, Loveman E, Mclntyre L, Waugh N: The effectiveness of diagnostic tests for the assessment of shoulder pain due to soft tissue disorders: a systematic review. Health Technol Assess 2003, 7:iii, l-iiil 66.

27. Green S, Buchbinder R, Glazier R, Forbes A: Systematic review of randomised controlled trials of interventions for painful shoulder: selection criteria, outcome assessment, and efficacy. BMJ 1998, 3 1 6:354-360.

28. van der Heijden GJ, van der Windt DA, de Winter AF: Physiotherapy for patients with soft tissue shoulder disorders: a systematic review of randomised clinical trials. BMJ I997, 3 I 5:25-30. 
29. Kirkley A, Alvarez C, Griffin S: The development and evaluation of a disease-specific quality-of-life questionnaire for disorders of the rotator cuff: The Western Ontario Rotator Cuff Index. Clin J Sport Med 2003, I 3:84-92.

30. Lo IK, Griffin S, Kirkley A: The development of a disease-specific quality of life measurement tool for osteoarthritis of the shoulder: The Western Ontario Osteoarthritis of the Shoulder (WOOS) index. Osteoarthritis Cartilage 2001, 9:771-778.

31. Roach KE, Budiman-Mak E, Songsiridej N, Lertratanakul Y: Development of a shoulder pain and disability index. Arthritis Care Res 1991, 4:143-149.

32. Beaton DE, Katz JN, Fossel AH, Wright JG, Tarasuk V, Bombardier C: Measuring the whole or the parts? Validity, reliability, and responsiveness of the Disabilities of the Arm, Shoulder and Hand outcome measure in different regions of the upper extremity. J Hand Ther 200 I, I 4: I28-I46.

33. Solway S, Beaton DE, McConnell S, Bombardier C: The Dash Outcome Measure User's manual 2nd edition. Toronto, Ont, Institute for Work and Health; 2002.

34. Geertzen JHB, Dijkstra PU, van-Sonderen ELP, Groothoff JW, tenDuis HJ: Relationship between imapirments, disability and handicap in reflex sympathetic dystrophy. Clin Rehabil 1998, 12:402-412.

35. Hirsch R, Guralnik JM, Leveille SG, Simonsick EM, Ling S, BandeenRoche K, Rantanen T, Pahor M, Fried LP, Hochberg MC: Severity of hand osteoarthritis and its association with upper extremity impairment in a population of disabled older women: the Women's Health and Aging Study. Aging (Milano) 1999, I I:253-26I.

36. MacDermid JC, Donner A, Richards RS, Roth JH: Patient versus injury factors as predictors of pain and disability six months after a distal radius fracture. J Clin Epidemiol 2002, 55:849-854.

37. Massy-Westropp N, Krishnan J, Ahern M: Comparing the AUSCAN Osteoarthritis Hand Index, Michigan Hand Outcomes Questionnaire, and Sequential Occupational Dexterity Assessment for patients with rheumatoid arthritis. J Rheumatol 2004, 3 I: | 996-200|.

38. Bostrom C, Harms-Ringdahl K, Nordemar R: Relationships between measurements of impairment, disability, pain, and disease activity in rheumatoid arthritis patients with shoulder problems. Scand J Rheumatol 1995, 24:352-359.

39. Michener LA: Relationships between impairments, threedimensional kinematics, functional limitation, and disability in patients with subacromial impingement syndrome. (MCP Hahnemann University) ** 2001; Ph D I75 p.:

40. Mannerkorpi K, Svantesson U, Broberg C: Relationships between performance-based tests and patients' ratings of activity limitations, self-efficacy, and pain in fibromyalgia. Arch Phys Med Rehabil 2006, 87:259-264.

41. MacDermid JC, Mule M: Concurrent validity of the NK hand dexterity test. Physiother Res Int 200I, 6:83-93.

42. Mathiowetz V: Validity of the Jebsen Hand Function test. Journal of Hand Therapy 1993, 6:65-66.

43. Spaulding SJ, McPherson JJ, Strachota E, Kuphal M, Ramponi M: Jebsen Hand Function Test: performance of the uninvolved hand in hemiplegia and of right-handed, right and left hemiplegic persons. Arch Phys Med Rehabil 1988, 69:419-422.

44. Stamm TA, Ploner A, Machold KP, Smolen J: Moberg picking-up test in patients with inflammatory joint diseases: a survey of suitability in comparison with button test and measures of disease activity. Arthritis Rheum 2003, 49:626-632.

45. Turgeon TR, MacDermid JC, Roth JH: Reliability of the NK dexterity board. J Hand Ther 1999, I2:7-I5.

46. van Lankveld W, van't Pad Bosch P, Bakker J, Terwindt S, Franssen M, van Riel P: Sequential Occupational Dexterity Assessment (SODA): a new test to measure hand disability. J Hand Ther 1996, 9:27-32

47. Hughes RE, Johnson ME, Skow A, An KN, O'Driscoll SW: Reliability of a Simple Shoulder Endurance Test. Journal of Musculoskeletal Research 1999, 3:195-200.

48. Barnes WS: The relationship between maximum isokinetic strength and isokinetic endurance. Res Quart 1980, 5 I:714-717.

49. Motzkin NE, Cahalan TD, Morrey BF, An K, Chao EYS: Isometric and isokinetic endurance testing of the forearm complex. American Journal of Sports Medicine, 19(2):I07-II, 199I Mar-Apr (I2 ref) 1991, I9:107-III.
50. Ozcakar L, Inanici F, Kaymak B, Abali G, Cetin A, Hascelik Z: Quantification of the weakness and fatigue in thoracic outlet syndrome with isokinetic measurements. Br J Sports Med 2005, 39: |78-|8|.

5I. Chandler TJ, Kibler WB, Stracener EC, Ziegler AK, Pace B: Shoulder strength, power, and endurance in college tennis players. $\mathrm{Am}$ J Sports Med 1992, 20:455-458.

52. Sharma S, Schumacher HR, McLellan TL: Evaluation of the Jebson hand function test for use in patients with rheumatoid arthritis. Arth Care Res 1994, 7:16-19.

53. van Lankveld WG, Graff MJ, PJ PB: The Short Version of the Sequential Occupational Dexterity Assessment based on individual tasks' sensitivity to change. Arthritis Care Res 1999 , 1 2:4 17-424.

54. Jebson RH, Taylor N, Trieschmann RB, Trotter MJ, Howard LA: An objective and standardized test of hand function. Arch Phys Med Rehabil 1969, 50:311-319.

55. Yanai T, Fuss FK, Fukunaga T: In vivo measurements of subacromial impingement: Substantial compression develops in abduction with large internal rotation. Clin Biomech (Bristol, Avon ) 2006

56. Struhl S: Anterior internal impingement: An arthroscopic observation. Arthroscopy 2002, I 8:2-7.

57. Calis M, Akgun K, Birtane M, Karacan I, Calis H, Tuzun F: Diagnostic values of clinical diagnostic tests in subacromial impingement syndrome. Ann Rheum Dis 2000, 59:44-47.

58. Beaton DE, Katz JN, Fossel AH, Wright JG, Tarasuk V, Bombardier C: Measuring the whole or the parts? Validity, reliability, and responsiveness of the Disabilities of the Arm, Shoulder and Hand outcome measure in different regions of the upper extremity. J Hand Ther 200।, | 4:|28-|46.

59. Williams JW Jr., Holleman DR Jr., Simel DL: Measuring shoulder function with the Shoulder Pain and Disability Index. J Rheumatol 1995, 22:727-732.

60. MacDermid JC, Solomon P, Prkachin K: The Shoulder Pain and Disability Index demonstrates factor, construct and longitudinal validity. BMC Musculoskelet Disord 2006, 7:12.

61. Shrout PE, Fleiss JL: Intraclass correlations: Uses in assessing rater reliability. Psychol Bull 1979, 86:420-428.

62. Meislin RJ, Sperling JW, Stitik TP: Persistent shoulder pain: epidemiology, pathophysiology, and diagnosis. Am J Orthop 2005, 34:5-9.

63. Ostor AJ, Richards CA, Prevost AT, Speed CA, Hazleman BL: Diagnosis and relation to general health of shoulder disorders presenting to primary care. Rheumatology (Oxford) 2005, 44:800-805.

\section{Pre-publication history}

The pre-publication history for this paper can be accessed here:

\section{http://www.biomedcentral.com/1471-2474/8/42/prepub}

Publish with Bio Med Central and every scientist can read your work free of charge

"BioMed Central will be the most significant development for disseminating the results of biomedical research in our lifetime. "

Sir Paul Nurse, Cancer Research UK

Your research papers will be:

- available free of charge to the entire biomedical community

- peer reviewed and published immediately upon acceptance

- cited in PubMed and archived on PubMed Central

- yours - you keep the copyright 